\title{
Trametinib: a MEK inhibitor for management of metastatic melanoma
}

This article was published in the following Dove Press journal:

OncoTargets and Therapy

25 August 2015

Number of times this article has been viewed

\author{
Iwona Lugowska',2 \\ Hanna Koseła-Paterczyk' \\ Katarzyna Kozak' \\ Piotr Rutkowski' \\ 'Department of Soft Tissue/Bone \\ Sarcoma and Melanoma, Maria \\ Sklodowska-Curie Memorial Cancer \\ Centre and Institute of Oncology, \\ 2Department of Epidemiology, \\ Institute of Mother and Child, \\ Warsaw, Poland
}

\begin{abstract}
This review presents the current data on the efficacy and safety of the selective mitogen-activated extracellular signal-regulated kinase (MEK) inhibitor trametinib in patients with metastatic BRAF V600-positive melanoma. The pharmacological, safety, and efficacy data come from the Phase I, II, and III studies of trametinib monotherapy, as well as those in combination with the BRAF inhibitor dabrafenib. The most common adverse effects of trametinib therapy are rash, dermatitis, diarrhea, and fatigue. The Phase III METRIC study showed significant improvement in overall survival and progression-free survival in favor of trametinib over standard dacarbazine or paclitaxel chemotherapy. Therefore, trametinib was approved by the US Food and Drug Administration and European Medicines Agency as a single agent for the treatment of patients with V600E-mutated metastatic melanoma. Progression-free survival and response rates for trametinib monotherapy were lower than those noted with BRAF inhibitors. The second step in developing trametinib was to use the combination of trametinib with the BRAF inhibitor, eg, dabrafenib, to postpone the progression on MEK or BRAF inhibitors. The recently published data showed significant improvement in overall survival and progression-free survival in favor of the combination of trametinib and dabrafenib over vemurafenib therapy or dabrafenib alone, with good tolerance. The US Food and Drug Administration has approved the combination of dabrafenib (150 mg orally twice daily) and trametinib (2 mg orally once daily) for the treatment of patients with $B R A F V 600 E / K$-mutant metastatic melanoma, and their use seems to be currently the best approach. While BRAF-MEK inhibition is a standard, molecular targeted therapy in $B R A F$-mutated melanomas, its future utility has to be established in the rapidly changing landscape of immunotherapeutics.
\end{abstract}

Keywords: trametinib, MEK inhibitor, dabrafenib, melanoma, BRAF mutation

\section{Introduction}

The mitogen-activated protein kinase (MAPK) signaling cascade plays a critical role in the transduction of extracellular signals to cellular responses. Four major subgroups within the MAPK family have been identified: extracellular signalregulated kinases (ERKs), c-jun N-terminal kinases, ERK5, and the p38 group of protein kinases (Figure 1). ${ }^{1}$ The Ras-regulated RAF/MEK/ERK pathway is known to regulate key cellular functions, including proliferation, survival, differentiation, angiogenesis, and migration. ${ }^{2}$ Impaired activation of the RAF/MEK/ERK pathway is common in melanoma. $B R A F$ (v-raf murine sarcoma viral oncogene homolog $\mathrm{B} 1$ ) and NRAS (neuroblastoma RAS viral [v-ras] oncogene homolog) mutations are found in $40 \%-60 \%$ and $10 \%-20 \%$ of cutaneous melanomas, respectively. ${ }^{3,4}$ The occurrence of these activated mutants is in general mutually exclusive. ${ }^{5}$ Unlike $B R A F$ mutations, activating mutations in $M E K$ are very rare, ${ }^{6}$ nevertheless, mitogen-activated extracellular signal-regulated kinase (MEK) activity appears to be critical for mutant BRAF
Department of Soft Tissue/Bone Sarcoma and Melanoma, Maria Sklodowska-Curie Memorial Cancer Center and Institute of Oncology, Roentgen Street 5, 02-78I Warsaw, Poland

Tel +48225462060

Fax +482 26439791

Email iwonalugowska@coi.pl 


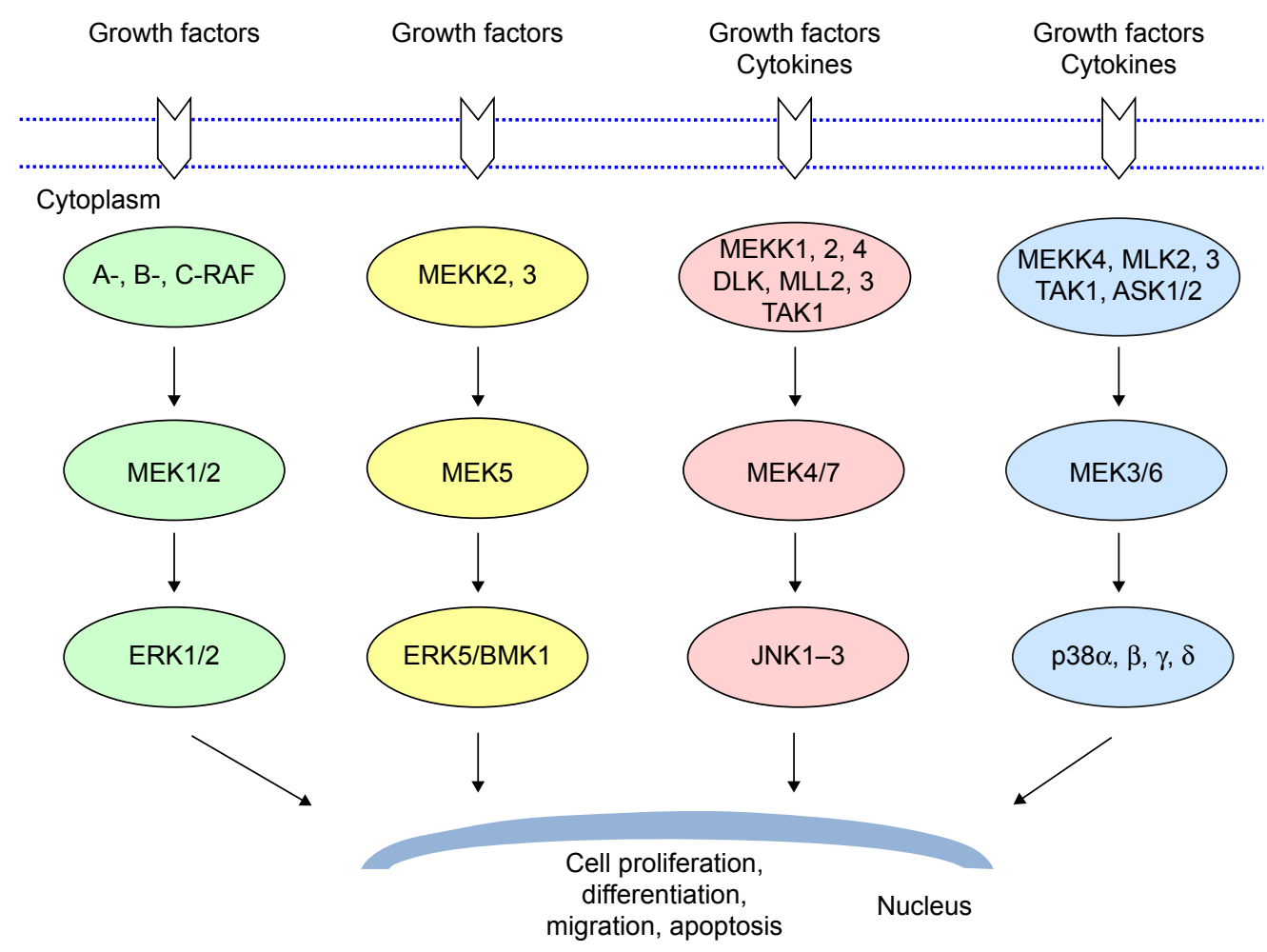

Figure I Mitogen-activated protein kinase signaling pathways.

signaling, since ERKs seem to be the only catalytic substrates for both MEK isoforms. ${ }^{1,7}$ In preclinical models of human melanoma, selective MEK inhibitors have inhibited growth and induced cell death in tumors bearing either $B R A F$ or NRAS mutations. ${ }^{8}$

Trametinib (Mekinist ${ }^{\mathrm{TM}}$ ) is a reversible and highly selective allosteric inhibitor of MEK1 and MEK2 with anticancer activity against metastatic melanoma carrying the $B R A F$ V600 mutation. Trametinib activity has been evaluated in the treatment of variety of cancers, and is currently approved as a monotherapy for subjects with unresectable or metastatic melanoma with BRAF V600E/K mutation, or in combination with dabrafenib for the same indication. ${ }^{9}$

\section{Review of pharmacology, mode of action, pharmacokinetics}

Trametinib is an orally available, small molecule, selective, and adenosine triphosphate-noncompetitive inhibitor of activation and kinase activity of MEK1 and MEK2 (also known as MAP2K1 and MAP2K2). The specificity of trametinib for MEK $1 / 2$ was confirmed against a panel of more than 180 kinases, including B-Raf, C-Raf, and the closest kinase homolog MEK5. ${ }^{10}$ Trametinib inhibited proliferation of BRAF V600E melanoma cell lines at concentrations of $1.0-2.5 \mathrm{nmol} / \mathrm{L} .{ }^{11}$ In xenografted tumor models, trametinib showed sustained inhibition of ERK phosphorylation, suppression of Ki67, and growth inhibition in tumor lines with mutant $B R A F$ or $R A S .{ }^{10}$ By these mechanisms, trametinib decreases cell proliferation, causes G1 cell cycle arrest, and induces apoptosis. ${ }^{11}$ The half maximum inhibitory concentration is $0.7-0.9 \mathrm{nmol} / \mathrm{L}$ for these enzymes. ${ }^{10,12} \mathrm{In}$ human subjects with solid tumors, trametinib pharmacokinetics were determined after single-repeat and repeat-dose oral administration of tablets with doses up to $2 \mathrm{mg}$ once daily under fasting conditions, at least 1 hour prior to or 2 hours after a meal. Trametinib is absorbed rapidly, with the median time to maximum plasma concentration occurring 1.5 hours after a single oral administration under fasting conditions. The absolute oral bioavailability of a single trametinib $2.0 \mathrm{mg}$ tablet is moderate to high $(72 \%)$ relative to a coadministered intravenous microdose. The drug accumulates with repeat dosing, with a mean accumulation ratio of 5.97 at the recommended dose of $2 \mathrm{mg}$ once daily and a terminal half-life of 5.3 days determined after administration of a single dose. Trametinib has a low drug extraction ratio based on a plasma intravenous clearance of $3.21 \mathrm{~L} / \mathrm{h}$, which represents approximately $1 \%$ of liver blood flow. It is an inducer of cytochrome P450 (CYP) 3A4; however, it is unlikely that this drug affects the pharmacokinetics of other drugs, because it is not an inhibitor of CYP1A2, CYP2A6, 
CYP2B6, CYP2D6, or CYP3A4. Therefore, there is a low likelihood of drug-drug interactions during trametinib use. Biochemical and cellular activities of trametinib and its metabolite demonstrated that both compounds are similarly active at inhibiting BRAFV600E-mediated MEK1 activation, phospho-MEK1 activity, cellular phosphorylation of ERK, and cellular proliferation of SK-MEL-28. When compared with other agents in this class, trametinib has a favorable pharmacokinetic profile, with a prolonged halflife, low peak-to-trough ratio, and limited toxicity. ${ }^{7,12,13}$ The clinical use of both BRAF and MEK inhibitors provides an additional therapeutic advantage, as they are able to control the dormancy of putative prometastatic disseminated tumor cells. Administration of dabrafenib $150 \mathrm{mg}$ twice daily in combination with trametinib $2 \mathrm{mg}$ once daily had little impact on the maximum plasma concentration of dabrafenib or the area under the concentration-time curve.

\section{Efficacy studies}

\section{Phase I studies of trametinib in monotherapy and in combination}

The development of trametinib (GSK-1120212) under the Investigational New Drug program was initiated in May 2008. MEK111054 was a Phase I, open-label, multiple-dose, dose-escalation, first-in-human study that investigated the safety, pharmacokinetics, and pharmacodynamics of the MEK inhibitor trametinib in subjects with solid tumors or lymphomas. ${ }^{12}$ A dose of $2.0 \mathrm{mg}$ administered once daily was selected for further studies based on tolerability, the exposureresponse relationship with pharmacodynamic markers in tumor biopsies, and clinical activity. An additional part of the study was dedicated only to melanoma patients. Thirty of 36 patients with $B R A F$ mutations had not received a BRAF inhibitor before. Two complete responses and ten partial responses were noted in this subgroup (confirmed response rate 33\%). Median progression-free survival (PFS) in this subgroup was 5.7 months (95\% confidence interval [CI] 4.0-7.4). One unconfirmed partial response was recorded in the group of six patients who had previously received a BRAF inhibitor. In the group of 39 patients with wild-type $B R A F$ melanoma, four partial responses were confirmed (confirmed response rate, $10 \%)^{13}$

Several Phase I studies have been conducted with trametinib in combination. The MEK112111 study showed no evidence of altered exposure for trametinib or gemcitabine; however, the addition of trametinib may increase gemcitabineassociated myelosuppression. Of ten patients with measurable pancreatic cancer, three partial responses (30\%) were documented; in addition, two patients achieved objective responses (breast, complete response; salivary glands, partial response). ${ }^{14}$ A Phase Ib study (MEK112110) investigated the safety and tolerability of trametinib in combination with everolimus, a mammalian target of rapamycin inhibitor, in patients with advanced solid tumors. Pharmacokinetic assessment did not suggest drug-drug interactions between the two agents; however, concurrent treatment resulted in mucosal inflammation (40\%), stomatitis (25\%), fatigue $(54 \%)$, and diarrhea (42\%). Of the 67 enrolled patients, five (7\%) achieved a partial response and $21(31 \%)$ had stable disease. Of the 21 patients with pancreatic cancer, one patient $(5 \%)$ had a partial response and six patients $(29 \%)$ had stable disease. Unfortunately, this study was unable to identify a recommended Phase II dose of trametinib in combination with everolimus that provided acceptable tolerability and adequate drug exposure. ${ }^{15}$ In another open-label, dose-finding Phase Ib study in patients with $R A S$-mutant or $B R A F$-mutant non-small cell lung, ovarian, or pancreatic cancer, use of buparlisib, a pan-phosphatidylinositide 3-kinase inhibitor, at a dose of 60 mg with trametinib $1.5 \mathrm{mg}$ daily showed promising antitumor activity in patients with $K R A S$-mutant ovarian cancer. Longterm tolerability of the combination is challenging, because its use requires frequent dose interruptions and reductions for toxicity. ${ }^{16}$ Trametinib also demonstrated preclinical and clinical activity in $K R A S$-mutant non-small cell lung cancer. MEK114653 was an open-label, multicenter, randomized Phase II study assessing the efficacy and safety of trametinib compared with docetaxel in second-line treatment of patients with locally advanced or metastatic non-small cell lung cancer with targeted mutations ( $K R A S, N R A S, B R A F, M E K 1)$. The study was prematurely terminated after the interim analysis, which showed the comparison of trametinib versus docetaxel for PFS crossed the futility boundary. ${ }^{17}$ In a Phase I study of the MEK inhibitor trametinib in combination with the AKT inhibitor afuresertib in patients with solid tumors and multiple myeloma, continuous daily dosing of the trametinibafuresertib combination was poorly tolerated, and further study of an intermittent dose schedule or combination of trametinib with more selective inhibitors may be warranted. ${ }^{18}$ In recently published data from PACMEL, a Phase 1 doseescalation trial of trametinib in combination with paclitaxel in advanced melanoma patients, six (40\%) partial responses were reported, including in four of eight patients with NRAS mutations; median PFS was 5.5 months and median overall survival was 14.1 months. In this small group, promising PFS and overall survival rates were observed in patients with melanoma lacking the BRAF V600 mutation. ${ }^{19}$ 


\section{Phase II studies of trametinib in monotherapy and in combination}

MEK113583 was an open-label, multicenter Phase II study investigating the objective response rate, safety, and pharmacokinetics of trametinib $2.0 \mathrm{mg}$ once daily in subjects with $B R A F$ mutation-positive melanoma, who had either failed previous therapy with a BRAF inhibitor (cohort A) or were treatment-naive for a BRAF inhibitor (cohort B). In cohort A $(n=40)$, minimal clinical activity was observed, with eleven patients (28\%) having stable disease; the median PFS was 1.8 months. In cohort B $(n=57)$, there was one $(2 \%)$ complete response, 13 (23\%) partial responses, and 29 patients (51\%) with stable disease (confirmed response rate, 25\%); the median PFS was 4.0 months. The activity of trametinib was observed in BRAF V600E mutants as well as in cases of less frequent mutations (BRAF K601E, BRAF V600R). The most common treatment-related adverse events for all patients were skin-related toxicity, nausea, peripheral edema, diarrhea, pruritus, and fatigue. No cutaneous squamous cell carcinoma was observed. These data supported further evaluation of trametinib in BRAF inhibitor-naive $B R A F$ mutant melanoma, including rarer forms of $B R A F$-mutant melanoma. ${ }^{20}$

Although the use of trametinib demonstrates significant clinical activity in patients with $B R A F$-mutated metastatic melanoma, most develop mechanisms of acquired resistance due to intrinsic resistance and MAPK-reactivating changes, and experience progression within 4-5 months following the initial treatment. A similar phenomenon is observed for monotherapy with BRAF inhibitors (dabrafenib or vemurafenib), although here the median PFS is slightly longer than for MEK inhibitors. ${ }^{21}$ It was believed that in order to avoid this unfavorable event, a multistage inhibition of the MAPK pathway was mandatory, providing the preclinical rationale for the combination of a BRAF-MEK inhibitor.

In the Phase I/II study, 162 patients with advanced melanoma and BRAF V600E/K mutations were randomized to three arms: a combination of dabrafenib $150 \mathrm{mg}$ twice daily and trametinib $1 \mathrm{mg}$ once daily; a combination of dabrafenib $150 \mathrm{mg}$ twice daily and trametinib $2 \mathrm{mg}$ once daily; and single-agent dabrafenib $150 \mathrm{mg}$ twice daily. ${ }^{22}$ Based on the results, the combination $150 \mathrm{mg}$ of dabrafenib twice daily plus $2 \mathrm{mg}$ of trametinib once daily was shown to be superior (objective response rate $76 \%$ versus $54 \% ; P=0.03$ ); the median duration of response was 10.5 months, compared with 5.6 months on monotherapy. The investigator-assessed median PFS with the combination was 9.4 months and 5.8 months for dabrafenib monotherapy (hazard ratio [HR]
0.39; 95\% CI $0.25-0.62 ; P<0.001)$. Approximately $80 \%$ of patients with disease progression crossed over from the monotherapy group to the combination arm. Patients in the combination group $(150 \mathrm{mg} / 2 \mathrm{mg})$ had significantly higher rates of complete response or partial response $(P=0.03)$ as compared with the monotherapy group ( $76 \%$ versus $54 \%$, respectively) and a longer median duration of response (10.5 months versus 5.6 months).

Another open-label Phase I/II study was dedicated to advanced melanoma patients after disease progression with BRAF inhibitor treatment administered before study enrollment, or after crossover at progression with dabrafenib monotherapy. The combination had modest clinical efficacy, especially in patients with BRAF inhibitor-resistant melanoma, who benefited from BRAF monotherapy for less than 6 months. ${ }^{23}$

\section{Phase III studies of trametinib in monotherapy and in combination}

\section{Trametinib monotherapy}

METRIC (MEK114267) was a Phase III, randomized, openlabel, multisite study comparing the use of trametinib with chemotherapy (dacarbazine or paclitaxel) in 322 subjects with advanced or metastatic BRAF V600E/K mutationpositive melanoma. Patients were randomized in a 2:1 ratio to receive trametinib $2 \mathrm{mg}$ once daily or chemotherapy, and were allowed to cross over from the chemotherapy arm to the trametinib arm in case of disease progression. Median PFS was 4.8 months in the trametinib group and 1.5 months in the chemotherapy group (HR 0.45 for disease progression or death in the trametinib group; 95\% CI 0.33-0.63; $P<0.001$ ). At 6 months, the rate of overall survival was $81 \%$ in the trametinib group and $67 \%$ in the chemotherapy group despite crossover (HR for death $0.54 ; 95 \%$ CI $0.32-0.92 ; P=0.01$ ). The objective response rates according to Response Evaluation Criteria In Solid Tumors criteria by investigator assessment were $22 \%$ for patients treated with trametinib and $8 \%$ for patients treated with chemotherapy $(P=0.001)$. The results of this registration study led to approval of trametinib at the dose of $2 \mathrm{mg}$ orally once daily as a single agent for treatment of patients with unresectable or metastatic melanoma with BRAF V600E or V600K mutations. ${ }^{24}$

\section{Combination of BRAF and MEK inhibitors}

In the Phase III trial (COMBI-d), 423 previously untreated melanoma patients with unresectable or metastatic disease harboring a BRAF V600E or $V 600 \mathrm{~K}$ mutation were 
randomized to receive a combination of dabrafenib $150 \mathrm{mg}$ orally twice daily and trametinib $2 \mathrm{mg}$ orally once daily or dabrafenib (and placebo). The median PFS was 9.3 months in the combination arm and 8.8 months in the dabrafenib only arm (HR $0.75 ; 95 \%$ CI $0.57-0.99 ; P=0.03$ ). The overall response rate was $67 \%$ in the combination group and $51 \%$ in the dabrafenib only group $(P=0.002)$. The interim 5-month overall survival rate was $93 \%$ with dabrafenib and trametinib and $85 \%$ with dabrafenib alone (HR 0.63; 95\% CI 0.42-0.94; $P=0.02$ ). Toxicity was similar in the two groups except for pyrexia, which occurred more often in the combination arm than in the monotherapy arm (51\% versus $28 \%) .{ }^{25}$ In the second Phase III open-label trial (COMBI-v), 704 treatmentnaïve patients with a diagnosis of metastatic melanoma and $B R A F$ V600 mutation were randomly assigned to receive either a combination of dabrafenib $150 \mathrm{mg}$ twice daily and trametinib $2 \mathrm{mg}$ orally once daily or vemurafenib orally $960 \mathrm{mg}$ twice daily. At the interim overall survival analysis performed after $77 \%$ of the total number of events, the overall survival rate at 12 months was $72 \%$ (95\% CI 67-77) in the combination therapy group and 65\% (95\% CI 59-70) in the vemurafenib group (HR 0.69; 95\% CI 0.53-0.89; $P=0.005)$; median overall survival was not reached in the combination therapy group as compared with 17.2 months in the vemurafenib group. Median PFS was 11.4 months in the combination therapy group and 7.3 months in the vemurafenib group (HR 0.56; 95\% CI 0.46-0.69; $P<0.001$ ). The objective response rate was $64 \%$ in the combination arm and $51 \%$ in the vemurafenib group $(P<0.001)$. Toxicity was similar in the two groups. The study was stopped for efficacy in July $2014 .^{26}$

BRAF and MEK inhibitors are the first groups of agents that have improved all clinical efficacy endpoints, including response rate, PFS, and overall survival, in patients diagnosed with $B R A F$-mutated advanced melanoma when compared with standard chemotherapy in randomized Phase III studies. The effect of trametinib in subjects with a variety of refractory cancers is currently under evaluation in ongoing clinical studies and at least 17 are completed.

A summary of outcomes for the Phase III trials of trametinib is presented in Table 1.

\section{Safety, tolerability, and quality of life: patient-focused perspectives}

In the randomized, Phase III open-label METRIC trial comparing the efficacy of trametinib with chemotherapy in first-line treatment of patients with metastatic $B R A F$-mutated melanoma, adverse events leading to dose interruptions in the trametinib group were observed in $35 \%$ of patients and leading to dose reductions in $27 \%{ }^{24}$ Two patients in this

Table I Summary of outcomes of Phase III trials of trametinib in monotherapy and in combination with dabrafenib for the treatment of BRAF V600-mutated melanoma

\begin{tabular}{|c|c|c|c|c|c|c|}
\hline & \multicolumn{2}{|l|}{ METRIC $^{24}$} & \multicolumn{2}{|l|}{ COMBI-d ${ }^{25,37}$} & \multicolumn{2}{|l|}{ COMBI-v² } \\
\hline & $\begin{array}{l}\text { Trametinib } \\
n=214\end{array}$ & $\begin{array}{l}\text { Chemotherapy } \\
n=108\end{array}$ & $\begin{array}{l}\text { Dabrafenib + } \\
\text { trametinib } \\
n=210\end{array}$ & $\begin{array}{l}\text { Dabrafenib } \\
n=210\end{array}$ & $\begin{array}{l}\text { Dabrafenib + } \\
\text { trametinib } \\
n=35 \text { I }\end{array}$ & $\begin{array}{l}\text { Vemurafenib } \\
n=350\end{array}$ \\
\hline CR, n (\%) & $4(2)$ & 0 & $22(10)$ & $18(9)$ & $47(13)$ & $27(8)$ \\
\hline PR, n (\%) & $43(20)$ & $9(8)$ & II 8 (56) & $90(43)$ & $179(5 \mid)$ & $153(44)$ \\
\hline SD & $119(56)$ & $34(31)$ & $54(26)$ & $59(33)$ & $92(26)$ & $106(30)$ \\
\hline RR, n (\%) & $22(16.6-28.1)$ & $8(3.9-15.2)$ & 67 & 51 & $\begin{array}{l}226(64) \\
(59.1-69.4)\end{array}$ & $\begin{array}{l}180(5 \mid) \\
(46 . \mid-56.2)\end{array}$ \\
\hline$P$-value & $<0.001$ & & $<0.001$ & & $<0.001$ & \\
\hline $\begin{array}{l}\text { Median duration } \\
\text { of response, months }\end{array}$ & NR & 5.5 & 10.2 & 9.2 & I3.8 (I I-NR) & $7.5(7.3-9.3)$ \\
\hline $\begin{array}{l}\text { Median PFS } \\
95 \% \mathrm{Cl}\end{array}$ & $4.8(4.3-4.9)$ & $1.5(1.4-2.7)$ & 9.3 & 8.8 & $11.4(9.9-14.9)$ & $7.3(5.8-7.8)$ \\
\hline $\mathrm{HR}(95 \% \mathrm{Cl})$ & $0.45(95 \% \mathrm{Cl} 0.33-0.63)$ & & $0.75(95 \%$ Cl 0.57-0.99) & & $0.56(0.46-0.69)$ & \\
\hline$P$-value & $<0.001$ & & 0.035 & & $<0.001$ & \\
\hline $\begin{array}{l}\text { OS rate (at } 6 \text { months) } \\
\text { (\%) }\end{array}$ & 81 & 67 & 93 & 85 & 72 & 65 \\
\hline $\mathrm{HR}(95 \% \mathrm{Cl})$ & $0.54(95 \% \mathrm{Cl} 0.32-0.92)$ & & $0.63(95 \% \mathrm{Cl} 0.42-0.94)$ & & $\begin{array}{l}0.69(95 \% \mathrm{Cl} \\
0.53-0.89)\end{array}$ & \\
\hline$P$-value & 0.01 & & 0.023 & & 0.005 & \\
\hline $\begin{array}{l}\text { Median OS, months } \\
(95 \% \mathrm{Cl})\end{array}$ & $15.6(14.0-17.4)$ & II.3 (7.2-14.8) & 25.1 & 18.7 & NR & 17.2 \\
\hline
\end{tabular}

Abbreviations: CR, complete response; PR, partial response; SD, stable disease; PFS, progression-free survival; OS, overall survival; HR, hazard ratio; NR, not reported; $\mathrm{RR}$, response rate; $\mathrm{Cl}$, confidence interval. 
group had serious grade 3 cardiac-related events that were considered to be drug-related, leading to permanent discontinuation of the study drug. There were no treatment-related deaths in this trial. The most common toxicities caused by MEK inhibition were rash, diarrhea, fatigue, and peripheral edema. The toxicities were managed with dose interruption and dose reductions (to $1.5 \mathrm{mg}$ or $1 \mathrm{mg}$ orally once daily). Secondary skin neoplasms were not observed. Adverse events that occurred in the trametinib arm are listed in Table 2. The most common treatment-related adverse events were rash/ dermatitis acneiform, diarrhea, and peripheral edema, which were also observed in the Phase I and Phase II studies with trametinib monotherapy. ${ }^{13,20}$ Rare but serious toxicities of trametinib, observed in patients treated in various clinical trials, are left ventricular dysfunction or decreased ejection fraction, pulmonary toxicity (interstitial pulmonary disease, pulmonary thrombosis), and ocular toxicities (central serous chorioretinopathy, retinal vein occlusion). ${ }^{13,14}$

The profile of skin toxicity observed while using MEK inhibitors differs from that occurring during treatment with BRAF inhibitors. Cutaneous squamous cell carcinoma, keratoacanthoma, or both, developed in approximately $18 \%$ of patients treated with vemurafenib ${ }^{27}$ and in approximately $10 \%$ of patients on dabrafenib; ${ }^{28}$ this type of secondary cutaneous neoplasm has not been reported during trametinib therapy. The nature of the rash observed with trametinib is papulopustular, and different to the hyperkeratotic maculopapular rash caused by vemurafenib. Another skin toxicity triggered by trametinib is acneiform eruption, and similar reactions have been reported with the use of epidermal growth factor receptor inhibitors like cetuximab. The eruptions do tend to appear on the face,

Table 2 Most common trametinib toxicities in the Phase III METRIC study

\begin{tabular}{|c|c|c|c|}
\hline \multirow[t]{2}{*}{$n=2 I \mid$} & Any grade* & Grade 2 & Grade 3 \\
\hline & \multicolumn{3}{|l|}{ Patients, n (\%) } \\
\hline Rash & $12 \mid(57)$ & $40(19)$ & $16(8) * *$ \\
\hline Diarrhea & $91(43)$ & $13(6)$ & 0 \\
\hline Fatigue & $54(26)$ & II (5) & $8(4)$ \\
\hline Peripheral edema & $54(26)$ & $8(4)$ & $2(1)$ \\
\hline Acneiform dermatitis & $40(19)$ & $20(9)$ & $2(1)$ \\
\hline Nausea & 38 (I8) & $5(2)$ & $2(1)$ \\
\hline Alopecia & $36(17)$ & $3(1)$ & $\mathrm{I}(<\mathrm{I})$ \\
\hline Arterial hypertension & $32(15)$ & $6(3)$ & $26(12)$ \\
\hline Constipation & $30(14)$ & $3(1)$ & 0 \\
\hline Vomiting & $27(13)$ & $3(1)$ & $2(1)$ \\
\hline
\end{tabular}

Notes: *Adverse events were graded on the basis of the Common Terminology Criteria for Adverse Events version 4.0. **In this group, there was one patient who experienced grade 4 rash. Reproduced from Flaherty KT, Robert C, Hersey P, et al. Improved survival with MEK inhibition in BRAF-mutated melanoma. N Engl J Med. 20I2;367(2):107-I|4. ${ }^{24}$ chest, and back, and this is likely to be due to the high number of sebaceous glands in these areas. Treatment of this form of skin toxicity usually includes topical antibiotics. ${ }^{29}$

Skin reactions occur within days after the treatment starts, and this rapid onset requires that patients have easy access to the treatment team, especially during the first few weeks of therapy. With close communication and dose adjustments, most severe reactions can be avoided and an optimal drug dose identified within the first 1-2 months of starting treatment. There are proposed schemes of treatment in the event of trametinib toxicity, involving (among others) temporary withdrawal of the drug potentially followed by a dose reduction. ${ }^{30}$ Situations requiring irreversible discontinuation are rare. ${ }^{31}$

The combination of dabrafenib and trametinib was tested in several clinical trials and randomized studies, which identified improved efficacy of the combination when compared with dabrafenib or vemurafenib alone. Interestingly, the combination regimen using full doses of both drugs generated fewer adverse skin events than BRAF inhibitor monotherapy, along with a lower frequency of malignant and hyperproliferative skin lesions. The lower incidence of squamoproliferative lesions during combined inhibition with BRAF/MEK is possibly related to blocking of paradoxical MAPK signaling downstream of CRAF by MEK inhibition. ${ }^{22,32}$ There were no new unexpected toxicities associated with the combination regimen, ${ }^{22,26}$ but when compared with a BRAF inhibitor alone, addition of trametinib adds rare toxicities related to the MEK inhibitor class, such as pyrexia, interstitial lung disease, venous thromboembolism, gastrointestinal bleeding, and heart (cardiomyopathy, decreased left ventricular ejection fraction) or ocular (retinal vein occlusion, uveitis) toxicity. The rates of permanent treatment discontinuation due to adverse events were similar for BRAF monotherapy and the BRAF/MEK combination (approximately 12\%), with pyrexia and decreased ejection fraction being the most common reasons in the combination therapy group. A summary of the most common adverse events in both Phase III trials of the combination of dabrafenib and trametinib is presented in Tables 3 and 4.

Last year, the results of a survey conducted during the METRIC study assessing quality of life for the first time during MEK inhibitor trials in metastatic melanoma patients were published. Quality of life was assessed at baseline and follow-up visits using the European Organization for Research and Treatment of Cancer core quality of life questionnaire. The study demonstrated that trametinib was associated with less functional impairment, smaller declines in health status, and less exacerbation of symptoms when compared with chemotherapy. ${ }^{33}$ The health-related quality of life assessment 
Table 3 Clinically significant adverse events occurring in $\geq 10 \%$ of patients in Phase III trials of the combination of dabrafenib and trametinib

\begin{tabular}{|c|c|c|c|c|c|c|c|c|}
\hline \multirow[t]{3}{*}{ AEs, n (\%) } & \multicolumn{4}{|l|}{ COMBI-v ${ }^{26}$} & \multicolumn{4}{|l|}{ COMBI- $^{25}$} \\
\hline & \multicolumn{2}{|c|}{$\begin{array}{l}\text { Dabrafenib + trametinib } \\
(\mathrm{n}=350)\end{array}$} & \multicolumn{2}{|c|}{$\begin{array}{l}\text { Vemurafenib } \\
(n=349)\end{array}$} & \multicolumn{2}{|c|}{$\begin{array}{l}\text { Dabrafenib + trametinib } \\
(\mathrm{n}=209)\end{array}$} & \multicolumn{2}{|l|}{$\begin{array}{l}\text { Dabrafenib } \\
(n=2 I I)\end{array}$} \\
\hline & All grades & Grade 3 & All grades & Grade 3 & All grades & Grade 3 & All grades & Grade 3 \\
\hline All events & $343(98)$ & $167(48)$ & 345 (99) & $198(57)$ & $199(95)$ & $66(32)$ & $203(96)$ & $72(34)$ \\
\hline Pyrexia & $184(53)$ & $15(4)$ & $73(21)$ & $2(<1)$ & $107(5 \mathrm{I})$ & $12(6)$ & $59(28)$ & $4(2)$ \\
\hline Nausea & $121(35)$ & $I(<I)$ & $125(36)$ & $2(<1)$ & $63(30)$ & 0 & $54(26)$ & $3(1)$ \\
\hline Diarrhea & $112(32)$ & $4(1)$ & $|3|(38)$ & $I(<I)$ & $51(24)$ & $\mathrm{I}(<\mathrm{I})$ & $30(14)$ & $2(<1)$ \\
\hline Chills & $110(3 \mathrm{I})$ & $3(<1)$ & $27(8)$ & 0 & $62(30)$ & 0 & $33(16)$ & 0 \\
\hline Fatigue & I0I (29) & $4(1)$ & $115(33)$ & $6(2)$ & $74(35)$ & $4(2)$ & $74(35)$ & $2(<\mathrm{I})$ \\
\hline Headache & I0I (29) & $3(<1)$ & $77(22)$ & $2(<1)$ & $63(30)$ & $\mathrm{I}(<\mathrm{I})$ & $62(29)$ & $3(1)$ \\
\hline Vomiting & $101(29)$ & $4(I)$ & $53(15)$ & $3(<1)$ & $42(20)$ & $2(<1)$ & $29(14)$ & $\mathrm{I}(<\mathrm{I})$ \\
\hline Arterial hypertension & $92(26)$ & $48(14)$ & $84(24)$ & $32(9)$ & $46(22)$ & $8(4)$ & $29(14)$ & $10(5)^{\prime}$ \\
\hline Arthralgia & $84(24)$ & $3(<1)$ & $\mid 78(5 \mid)$ & $15(4)$ & $5 I(24)$ & $I(<I)$ & $8(27)$ & 0 \\
\hline Rash & $76(22)$ & $4(1)$ & 149 (43) & $30(9)$ & $48(23)$ & 0 & $46(22)$ & $2(<\mathrm{I})$ \\
\hline Pruritus & $30(9)$ & 0 & $75(2 I)$ & $3(<1)$ & & & & \\
\hline Alopecia & $20(6)$ & 0 & 137 (39) & $I(<1)$ & $15(7)$ & 0 & $55(26)$ & 0 \\
\hline Hyperkeratosis & $15(4)$ & 0 & $86(25)$ & $2(<1)$ & $7(3)$ & 0 & $68(32)$ & $\mathrm{I}(<\mathrm{I})$ \\
\hline Photosensitivity & $13(4)$ & 0 & $78(22)$ & $I(<1)$ & & & & \\
\hline Skin papilloma & $6(2)$ & 0 & $80(23)$ & $2(<1)$ & $3(1)$ & 0 & $45(2 I)$ & 0 \\
\hline $\begin{array}{l}\text { Cutaneous SCC (including } \\
\text { keratoacanthoma) }\end{array}$ & $5(1)$ & & $63(18)$ & & $5(2)$ & & $20(9)$ & \\
\hline
\end{tabular}

Abbreviations: AEs, adverse events; SCC, squamous cell carcinoma.

based on the Phase III COMBI-d study demonstrated further that the combination of dabrafenib and trametinib is associated with better preservation of health-related quality of life and improvements in pain as compared with dabrafenib alone. ${ }^{34}$

\section{Conclusion and perspectives}

Trametinib is a novel oral MEK inhibitor with proven clinical activity in $B R A F(V 600)$-mutant metastatic melanoma alone and in combination with dabrafenib. ${ }^{11,14,15}$ The most common adverse effects associated with trametinib therapy are rash, dermatitis, diarrhea, and fatigue. The Phase III study MEK114267 (METRIC) showed significant improvement in overall survival and PFS in favor of trametinib over standard dacarbazine or paclitaxel chemotherapy. ${ }^{24}$

Trametinib was approved as a single agent for the treatment of patients with $V 600 E$-mutated metastatic melanoma by the US Food and Drug Administration in May 2013 and by the European Medicines Agency in September 2013. ${ }^{35} \mathrm{Nev}-$ ertheless, PFS and response rates for trametinib monotherapy was lower than those noted with BRAF inhibitors. The second step in the development of trametinib was its combination with a BRAF inhibitor, eg, dabrafenib, to postpone progression on MEK or BRAF inhibitors. The recently published data showed significant improvement in overall survival and PFS in favor of the combination of trametinib and dabrafenib over standard vemurafenib therapy ${ }^{26}$ or dabrafenib alone..$^{25}$ The combination is well tolerated with manageable side effects. As a result of this research, on January 8, 2014, the US Food and Drug Administration approved the combination of dabrafenib (150 mg orally twice daily) and trametinib ( $2 \mathrm{mg}$ orally once daily) for the treatment of patients with BRAF V600E/K-mutant metastatic melanoma. ${ }^{36}$ The aim

Table 4 Trametinib-related adverse events in Phase III trials of the combination of dabrafenib and trametinib

\begin{tabular}{|c|c|c|c|c|}
\hline AEs, n (\%) & $\begin{array}{l}\text { Dabrafenib + trametinib } \\
(n=350)\end{array}$ & $\begin{array}{l}\text { Vemurafenib } \\
(n=349)\end{array}$ & $\begin{array}{l}\text { Dabrafenib + trametinib } \\
(\mathrm{n}=209)\end{array}$ & $\begin{array}{l}\text { Dabrafenib } \\
(n=2 I I)\end{array}$ \\
\hline Diarrhea & $112(32)$ & |3| (38) & $51(24)$ & $30(14)$ \\
\hline Hypertension & $92(26)$ & $84(24)$ & $46(22)$ & $29(14)$ \\
\hline Acneiform dermatitis & $22(6)$ & $20(6)$ & $16(8)$ & $7(3)$ \\
\hline Decreased ejection fraction & $29(8)$ & 0 & $9(4)$ & $5(2)$ \\
\hline Chorioretinopathy & $2(<1)$ & $\mathrm{I}(<\mathrm{I})$ & $\mathrm{I}(<\mathrm{I})$ & $\mathrm{I}(<\mathrm{I})$ \\
\hline
\end{tabular}

Note: Adapted from Long GV, Stroyakovskiy D, Gogas $\mathrm{H}$, et al. Combined BRAF and MEK inhibition versus BRAF inhibition alone in melanoma. N Engl J Med. 20 I4;37I (20): 1877-1888, ${ }^{25}$ and Robert C, Karaszewska B, Schachter J, et al. Improved overall survival in melanoma with combined dabrafenib and trametinib. N Engl J Med. 20I5;372(I):30-39.26

Abbreviation: AEs, adverse events. 
of this combined blockade is to delay resistance occurring during BRAF or MEK monotherapy due to reactivation of the MAPK pathway, what prolongs patient survival, and to reduce the cutaneous toxicity related to paradoxical activation of the MAPK pathway in $B R A F$ wild-type keratinocytes, which is characteristic of the BRAF inhibitor class. The use of trametinib or the BRAF-MEK inhibitor combination after progression on a BRAF inhibitor is not effective, and currently the best approach is an upfront combination therapy, which became the standard targeted therapy in $B R A F$ mutated melanomas in the USA. In Europe, this therapeutic is still not formally approved. Recent data from the 2015 American Society of Clinical Oncology annual meeting confirmed the overall survival benefit from the trametinib and dabrafenib combination (a 29\% reduction in risk of death) in the COMBI-d study, with reported median overall survival of 25.1 months for the combination arm versus 18.7 months in the dabrafenib only arm and a 2-year overall survival rate exceeding 50\% for combined BRAF-MEK inhibitor therapy. ${ }^{37}$ While BRAF-MEK inhibition is a standard molecular targeted therapy in $B R A F$-mutated melanomas, its future utility has to be established in the rapidly changing landscape of immunotherapeutics (as immune checkpoint blockade using anti-cytotoxic T lymphocyte antigen-4 and anti-programmed death-1/programmed death-L1 antibodies).

Additionally, studies have shown that some patients with NRAS-mutated melanoma respond to MEK inhibitorbased therapy, ${ }^{38}$ what may extend the indication for MEK inhibitors in melanoma therapy in the future. Moreover, some preclinical studies imply that further investigation of the efficacy of trametinib in $B R A F / N R A S$ wild-type melanoma is warranted, ${ }^{39}$ and in combination with inhibition of phosphatidylinositide 3-kinase in uveal melanoma. ${ }^{40}$ The high activity of combined BRAF/MEK inhibition in terms of response rates, as well as accumulating data on the low number of newly developed keratoproliferative lesions, is leading to testing of these drugs in the neoadjuvant and adjuvant setting in high-risk melanomas.

\section{Disclosure}

PK has received honoraria and served as a member of advisory boards for GSK, Roche, Novartis, BMS, MSD, and Amgen. IL has received a travel grant from Novartis. The authors report no conflicts of interest in this work.

\section{References}

1. Montagut C, Settleman J. Targeting the RAF-MEK-ERK pathway in cancer therapy. Cancer Lett. 2009;283(2):125-134.

2. Akinleye A, Furqan M, Mukhi N, Ravella P, Liu D. MEK and the inhibitors: from bench to bedside. J Hematol Oncol. 2013;6:27.
3. Jiveskog S, Ragnarsson-Olding B, Platz A, Ringborg U. N-ras mutations are common in melanomas from sun-exposed skin of humans but rare in mucosal membranes or unexposed skin. J Invest Dermatol. 1998;111(5): 757-761.

4. Davies H, Bignell GR, Cox C, et al. Mutations of the BRAF gene in human cancer. Nature. 2002;417(6892):949-954.

5. Rutkowski P, Gos A, Jurkowska M, et al. Molecular alterations in clinical stage III cutaneous melanoma: correlation with clinicopathological features and patient outcome. Oncol Lett. 2014;8(1): $47-54$.

6. Samatar AA, Poulikakos PI. Targeting RAS-ERK signalling in cancer: promises and challenges. Nat Rev Drug Discov. 2014;13(12): 928-942.

7. Salama AK, Kim KB. MEK inhibition in the treatment of advanced melanoma. Curr Oncol Rep. 2013;15(5):473-482.

8. Solit DB, Garraway LA, Pratilas CA, et al. BRAF mutation predicts sensitivity to MEK inhibition. Nature. 2006;439(7074):358-362.

9. Rissmann R, Hessel MH, Cohen AF. Vemurafenib/dabrafenib and trametinib. Br J Clin Pharmacol. Epub 2015 April 6.

10. Yamaguchi T, Kakefuda R, Tajima N, Sowa Y, Sakai T. Antitumor activities of JTP-74057 (GSK1120212), a novel MEK1/2 inhibitor, on colorectal cancer cell lines in vitro and in vivo. Int J Oncol. 2011;39(1): 23-31.

11. Gilmartin AG, Bleam MR, Groy A, et al. GSK1120212 (JTP-74057) is an inhibitor of MEK activity and activation with favorable pharmacokinetic properties for sustained in vivo pathway inhibition. Clin Cancer Res. 2011;17(5):989-1000.

12. Infante JR, Fecher LA, Falchook GS, et al. Safety, pharmacokinetic, pharmacodynamic, and efficacy data for the oral MEK inhibitor trametinib: a Phase 1 dose-escalation trial. Lancet Oncol. 2012;13(8): 773-781.

13. Falchook GS, Lewis KD, Infante JR, et al. Activity of the oral MEK inhibitor trametinib in patients with advanced melanoma: a Phase 1 dose-escalation trial. Lancet Oncol. 2012;13(8):782-789.

14. Infante JR, Papadopoulos KP, Bendell JC, et al. A Phase $1 \mathrm{~b}$ study of trametinib, an oral mitogen-activated protein kinase kinase (MEK) inhibitor, in combination with gemcitabine in advanced solid tumours. Eur J Cancer. 2013;49(9):2077-2085.

15. Tolcher AW, Bendell JC, Papadopoulos KP, et al. A Phase IB trial of the oral MEK inhibitor trametinib (GSK1120212) in combination with everolimus in patients with advanced solid tumors. Ann Oncol. 2015;26(1): $58-64$.

16. Bedard PL, Tabernero J, Janku F, et al. A Phase Ib dose-escalation study of the oral pan-PI3K inhibitor buparlisib (BKM120) in combination with the oral MEK1/2 inhibitor trametinib (GSK1120212) in patients with selected advanced solid tumors. Clin Cancer Res. 2015;21(4): 730-738.

17. Blumenschein GR Jr, Smit EF, Planchard D, et al. A randomized Phase II study of the MEK1/MEK2 inhibitor trametinib (GSK1120212) compared with docetaxel in KRAS-mutant advanced non-small-cell lung cancer (NSCLC). Ann Oncol. 2015;26(5):894-901.

18. Tolcher AW, Patnaik A, Papadopoulos KP, et al. Phase I study of the MEK inhibitor trametinib in combination with the AKT inhibitor afuresertib in patients with solid tumors and multiple myeloma. Cancer Chemother Pharmacol. 2015;75(1):183-189.

19. Coupe N, Corrie P, Hategan M, et al. PACMEL: a Phase 1 dose escalation trial of trametinib (GSK1120212) in combination with paclitaxel. Eur J Cancer. 2015;51(3):359-366.

20. Kim KB, Kefford R, Pavlick AC, et al. Phase II study of the MEK1/ MEK2 inhibitor Trametinib in patients with metastatic BRAF-mutant cutaneous melanoma previously treated with or without a BRAF inhibitor. J Clin Oncol. 2013;31(4):482-489.

21. RutkowskiP, BlankC. Dabrafenib for the treatment of BRAF V600-positive melanoma: a safety evaluation. Expert Opin Drug Saf. 2014;13(9): 1249-1258.

22. FlahertyKT, Infante JR, Daud A, etal.CombinedBRAF and MEK inhibition in melanoma with BRAF V600 mutations. N Engl J Med. 2012;367(18): 1694-1703. 
23. Johnson DB, Flaherty KT, Weber JS, et al. Combined BRAF (dabrafenib) and MEK inhibition (trametinib) in patients with BRAFV600mutant melanoma experiencing progression with single-agent BRAF inhibitor. J Clin Oncol. 2014;32(33):3697-3704.

24. Flaherty KT, Robert C, Hersey P, et al. Improved survival with MEK inhibition in BRAF-mutated melanoma. $N$ Engl J Med. 2012; 367(2):107-114

25. Long GV, Stroyakovskiy D, Gogas H, et al. Combined BRAF and MEK inhibition versus BRAF inhibition alone in melanoma. $N$ Engl J Med. 2014;371(20):1877-1888.

26. Robert C, Karaszewska B, Schachter J, et al. Improved overall survival in melanoma with combined dabrafenib and trametinib. NEngl J Med. 2015;372(1):30-39.

27. Chapman PB, Hauschild A, Robert C, et al. Improved survival with vemurafenib in melanoma with BRAF V600E mutation. $N$ Engl J Med. 2011;364(26):2507-2516.

28. Hauschild A, Grob JJ, Demidov LV, et al. Dabrafenib in BRAF-mutated metastatic melanoma: a multicentre, open-label, Phase 3 randomised controlled trial. Lancet. 2012;380(9839):358-365.

29. Anforth R, Liu M, Nguyen B, et al. Acneiform eruptions: a common cutaneous toxicity of the MEK inhibitor trametinib. Australas J Dermatol. 2014;55(4):250-254.

30. Welsh SJ, Corrie PG. Management of BRAF and MEK inhibitor toxicities in patients with metastatic melanoma. Ther Adv Med Oncol. 2015;7(2): $122-136$.

31. Joshi L, Karydis A, Gemenetzi M, Shao EH, Taylor SR. Uveitis as a result of MAP kinase pathway inhibition. Case Rep Ophthalmol. 2013;4(3): 279-282.

32. King AJ, Arnone MR, Bleam MR, et al. Dabrafenib; preclinical characterization, increased efficacy when combined with trametinib, while BRAF/MEK tool combination reduced skin lesions. PLoS One. 2013;8(7):e67583.
33. Schadendorf D, Amonkar MM, Milhem M, et al. Functional and symptom impact of trametinib versus chemotherapy in BRAF V600E advanced or metastatic melanoma: quality-of-life analyses of the METRIC study. Ann Oncol. 2014;25(3):700-706.

34. Schadendorf D, Amonkar MM, Stroyakovskiy D, et al. Health-related quality of life impact in a randomised Phase III study of the combination of dabrafenib and trametinib versus dabrafenib monotherapy in patients with BRAF V600 metastatic melanoma. Eur $J$ Cancer. 2015;51(7):833-840.

35. Reuters. GSK melanoma drugs add to tally of U.S. drug approvals Available from: http://www.reuters.com/article/2013/05/30/us-glaxosmithkline-approvals-idUSBRE94S1A020130530. Accessed June 15, 2015.

36. OncLive. Dabrafenib/trametinib combination approved for advanced melanoma. January 9, 2014. Available from: http://www.onclive. com/web-exclusives/FDA-Approves-First-Ever-Combination-forMetastatic-Melanoma. Accessed June 15, 2015.

37. Long GV, Stroyakovskiy D, Gogas H, et al. Dabrafenib and trametinib versus dabrafenib and placebo for Val600 BRAF-mutant melanoma: a multicentre, double-blind, Phase 3 randomised controlled trial. Lancet. Epub 2015 May 29.

38. Ascierto PA, Schadendorf D, Berking C, et al. MEK162 for patients with advanced melanoma harbouring NRAS or Val600 BRAF mutations: a non-randomised, open-label Phase 2 study. Lancet Oncol. 2013;14(3): 249-256.

39. Ranzani M, Alifrangis C, Perna D, et al. BRAF/NRAS wild-type melanoma, NF1 status and sensitivity to trametinib. Pigment Cell Melanoma Res. 2015;28(1):117-119.

40. Khalili JS, Yu X, Wang J, et al. Combination small molecule MEK and PI3K inhibition enhances uveal melanoma cell death in a mutant GNAQ- and GNA11-dependent manner. Clin Cancer Res. 2012;18(16): 4345-4355.
OncoTargets and Therapy

\section{Publish your work in this journal}

OncoTargets and Therapy is an international, peer-reviewed, open access journal focusing on the pathological basis of all cancers, potential targets for therapy and treatment protocols employed to improve the management of cancer patients. The journal also focuses on the impact of management programs and new therapeutic agents and protocols on

\section{Dovepress}

patient perspectives such as quality of life, adherence and satisfaction. The manuscript management system is completely online and includes a very quick and fair peer-review system, which is all easy to use. Visit http://www.dovepress.com/testimonials.php to read real quotes from published authors. 